\title{
Control of flow geometry using electromagnetic body forcing
}

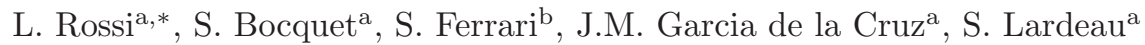 \\ ${ }^{a}$ Imperial College London, Department of Aeronautics, South Kensington Campus, SW7 \\ 2AZ, London, UK \\ ${ }^{b}$ Dipartimento di Ingegneria del Territorio, Universita' degli Studi di Cagliari
}

\begin{abstract}
This paper presents conceptual experiments and simulations aiming at controlling flow geometries. Such flow design is performed by driving electromagnetically a shallow layer of brine, the forcing being generated by a transverse electrical current and different combinations of permanent magnets placed underneath the brine supporting wall. It is shown how different basic flow characterisctics can be obtained with a single pair of magnets, by varying the angle with the electrical current. These basic flows are proposed as potential building blocks for advanced and complex flows studies. Three typical flow structures are presented to illustrate these building blocks. The discussion is then extended to multi-scale geometry by using blocks of various sizes. The flow is analysed using complementary experiments and numerical simulations. A good agreement is found between the 3D simulations and the experiments for both velocity and acceleration fields, which allows a higher degree of confidence in designing and modelling such flows. As the control of the flow geometry is important for mixing, in particular at low Reynolds number, we also illustrate the different stirring properties of the electromagnetically forced flows by comparing visualisations of passive scalars. They reveal complementary mixing properties for each of the building blocks.
\end{abstract}

Key words: DNS; PIV; Acceleration; Lorentz body forcing; electromagnetic; flow design; mixing; stirring

\section{Introduction}

Following the seminal work of $[6,15,9]$, electromagnetic forcing have been used in a variety of research areas, for different practical purposes: generation of chaotic and turbulent flows to study mixing, with and without chemical reactant $[27,4,10]$, drag reduction [5], flow separation delay [28], boundary layers manipulation [22] and heat transfers enhancement [12, 11]. At low Reynolds

*1.rossi@imperial.ac.uk 
number, such body forcing can be used to enhance mixing by manipulating the flow so as to generate turbulent like-features, e.g. [23, 14].

In the present work, electromagnetic forces are used to shape the flow field in a water tank. This paper is intended to present different arrangement of electromagnetic forces that can be used to generate different primitive flow patterns. These building elements are shown to produce different flow geometries and topologies each with different mixing properties. Combined in multi-scale forcing temporal schemes, they are amenable to generate very complex flows. The main objective of this paper is to analyse the properties of each arrangement, and then to combine them in a relatively simple multi-scale arrangement.

Efficient mixing can be achieved by designing flows and mixers to get chaotic and possibly turbulent-like properties. The control of the flow geometry and its temporal evolution is an important aspect of chaotic mixing, if not mixing in general. For example, [29] gives a brief introduction to the mathematical foundations of chaotic mixing and a Link Twist Maps (LTMs) approach where the spatio-temporal evolution of the flow geometry occupies a central place. Also, pertinent combination of stretching and folding produced by the temporal evolution and control of the flow geometry can lead to baker-like transformations and Smale horseshoe which produce exponential growth of material element lines and possibly interfaces, e.g. tendril-whorl [13] and blinking-vortex [3] flows. A complementary approach to chaotic mixing is to mimic the stirring of large-scale features, i.e. by forcing the flow to have turbulent-like properties. Recent works by the authors have partially addressed this issue by generating a new class of multi-scale laminar flow controlled by electromagnetic forcing (e.g. Rossi et al. $[23,24])$. The rationale is to produce a multi-scale flow where the scales are inserted in each other following an eight in eight pattern. This is performed by driving the flow with electromagnetic body forces generated by a multi-scale distribution of permanent magnets. An example is given on Fig. 1(c) by the pairs of North $(\mathrm{N})$ and South $(\mathrm{S})$ poles. The turbulent-like properties of the flow (e.g. power-law energy spectrum and Richardson-like diffusion [23, 24]) are solely controlled by the multi-scale distribution of the magnets and are similar to a fully turbulent flow field. It is important to point out that such flows are performed at Reynolds numbers of the order of unity, [14].

This paper has two main objectives. First it shows how particular flow topologies can be designed for such low Reynolds number flows and the geometry can be controlled using different configurations of the electromagnetic forcing. This approach is complementary to the critical-points concept (e.g. [18]) and to topological work on chaotic flows generated by electromagnetic forces [17]. By controlling the flow basic geometries, our intention is to isolate key elements of stirring (e.g. stretching, folding, permutation/rotation). This is achieved by controlling the nature (hyperbolic or elliptic) and the distribution of stagnation points along with the properties (intensity, strain) of their connection. The design and control of these key mechanism could then be applied to mixing studies. The second objective is to present an extensive comparison of experiments (e.g. acceleration measurements) and numerical simulations for different geometry of flow and forcing. Such comparison is relatively new and complements other re- 
cent work by $[14,1,12]$, also based on 3D simulations of detailed 3D models of the electromagnetic forces. Experimentally, velocity and acceleration fields are obtained at the free-surface using PIV, Particle tracking and PTVA algorithm [7]. Three-dimensional Direct Numerical Simulations (DNS) of the full flow field are performed, using a detailed model of electromagnetic forcing, close to the experimental conditions [14]. Even if numerous experimental studies of 2D turbulence based on electromagnetic forcing of a shallow layer of brine can be found in literature (e.g. [25, 20]), numerical simulation using a detailed model of electromagnetic forcing close to the experimental conditions are still rare, e.g. $[12,14,1]$. The use of a full 3D model of the flow configuration has significantly improved the accuracy of the simulations, compared to a $2 \mathrm{D}$ approach previously used [8]. However, some limitations (attributed to the modeling of the free surface) have been previously reported, most notably at high current intensity. Hence, the paper will focus mainly on low intensity.

Results for various flow geometries are reported in sections 3 and 4. First (section 3), a steady geometry of the forcing for a single pair of magnets is studied, for different angles between the current $j$ and the N-S axis. Second (section 4), an application to a more complex configuration, with a multi-scale distribution of magnets, is reported. In all cases, the different stirring properties of the flows are illustrated using visualisations based on the dispersion of passive scalar. By comparing and validating experiments and numerical simulations the authors intend to finally confirm that such electromagnetically forced flows can be properly performed and modeled in consistent 3D numerical simulations. This is firstly performed for the velocity fields. Then the comparison of the acceleration fields provide a deeper analysis as such fields are more sensitive to experimental conditions and models.

\section{Flow configurations, experiments and numerical approaches}

\subsection{Electromagnetically driven flows}

The flow in a shallow layer brine can be driven by an electromagnetic force $\mathbf{f}(\mathbf{x})$, with $\mathbf{f}=\mathbf{j} \times \mathbf{B}$, where $\mathbf{j}(\mathbf{x})$ is the electrical current density and $\mathbf{B}(\mathbf{x})$ is the magnetic field, given by the induction (1) and Ohm's law (2) equations, respectively:

$$
\begin{gathered}
\frac{\partial \mathbf{B}}{\partial t}=\nabla \times(\mathbf{u} \times \mathbf{B})+\frac{1}{\mu \sigma} \nabla^{2} \mathbf{B} \\
\mathbf{j}=\sigma(\mathbf{u} \times \mathbf{B}+\mathbf{E})
\end{gathered}
$$

where $\mathbf{u}(\mathbf{x}, t)$ is the velocity vector, $\mu$ is the magnetic permeability, $\sigma$ the electrical conductivity and $\mathbf{E}$ the imposed external electric field. If the conductivity $\sigma$ is low (as is the case here) and the velocity moderate, the magnetic Reynolds number, defined as $R e_{e m}=\sigma \mu u l$ is very small compared to 1 . Also, in the case of steady permanent magnets, $\frac{\partial \mathbf{B}}{\partial t}=0$. It follows that the magnetic field can be approximated as purely diffusive, and Eq. 1 reduces to:

$$
\nabla^{2} \mathbf{B}=0
$$


In addition, the induced electrical current $\sigma(\mathbf{u} \times \mathbf{B})$ can be neglected as $\frac{u B}{E} \sim$ $10^{-3}$. The electric density is then imposed only by the external electric field, $\mathbf{j}=$ $\sigma \mathbf{E}$, and the electromagnetic forces are consequently assumed to be independent of the flow velocity.

The Hartmann number $H a\left(H a^{2}=H^{2} f / \rho \nu u\right)$ is larger than one, for all flow considered hereafter, meaning that the electromagnetic forces are high enough to overcome the damping forces due to viscosity (essentially due to the bottom friction). In the present case, the forcing is not a perturbation of an existing flow: without the electromagnetic forcing, there is no flow.

Two different configurations are considered, both experimentally and numerically: a single pair of magnets (Fig. 1b) for different angle $\theta$, and a multiscale arrangement of magnets (Fig. 1c). The general setting, valid for both cases, is shown on Fig. 1(a), and in both setup, the magnetic induction of the permanent magnets is $B_{r}=0.68 T$, the salt concentration is $158 \mathrm{~g} / \mathrm{l}$, the viscosity $\nu$ is $1.3610^{-6} \mathrm{~m}^{2} \mathrm{~s}^{-1}$, the density $\rho=1105 \mathrm{kgm}^{-3}$ and its conductivity, $\sigma=16.6 \mathrm{Sm}^{-1}[30]$ and the magnetic permeability $\mu \simeq \mu_{0}=4 \pi 10^{-7} \mathrm{VsA}^{-1} \mathrm{~m}^{-1}$.

For the single pair of magnets, the two square permanent magnets are $40 \mathrm{~mm}$ aside. The electrical current density, $j=I / S$ where I is the electrical current and $S$ the cross section of the brine is kept constant at a value of about $j=$ $13.5 \mathrm{~A} / \mathrm{m}^{2}(I=48.6 \mathrm{~mA})$. This leads to an electromagnetic forcing of constant intensity with $f_{r m s}=3.26 \mathrm{Nm}^{-3}$.

For the multi-scale forcing the magnets sizes vary from $10 \mathrm{~mm}$ for the smallest scales to $160 \mathrm{~mm}$ for the two largest magnets (Fig. 1c, see [23] for more details on the multi-scale geometry). The flows is driven by electromagnetic forces of quasi-constant mean intensity within the brine above each magnets. The root mean square of the forces computed over the experimental domain is $f_{r m s}=$ $5.58 \mathrm{Nm}^{-3}$ when $j=62.4 \mathrm{~A} / \mathrm{m}^{2}(I=0.53 \mathrm{~A})$.

The thickness of the brine, $H$, is chosen as reference length scale: $x^{+}=$ $x / H, y^{+}=y / H, y^{+}=y / H$ and $L^{+}=L . / H$. The velocity and acceleration are normalised by the root mean square velocity and acceleration computed over the experimental domains. These dimensionless velocities and Lagrangian accelerations are noted $\mathbf{u}^{*}=\mathbf{u} / u_{r m s}$ and $\mathbf{a}^{*}=\mathbf{a} / a_{r m s}$.

The flow is initially at rest. When the current is switched on, the flow velocity increases until it reaches a quasi-steady state. Flow configurations, experiments and numerical simulations are compared only when this quasi-steady state is reached.

\subsection{Experiments}

Experiments are performed in two different tanks with driven flows (above the brine supporting horizontal walls) of size $\left(L_{x}, H, L_{z}\right)=(600 \mathrm{~mm}, 6 \mathrm{~mm}, 500 \mathrm{~mm})$ for a single pair of magnets, and of $\left(L_{x}, H, L_{z}\right)=(1700 \mathrm{~mm}, 5 \mathrm{~mm}, 1400 \mathrm{~mm})$ for the multi-scale distribution. The brine-supporting walls are checked so as to keep it straight and horizontal, with a standard deviation of the order of $0.1 \mathrm{~mm}$.

For the forcing with a single-pair of magnets, the pair of permanent magnets (both of $40 \mathrm{mmx} 40 \mathrm{mmx} 40 \mathrm{~mm}$ ) is placed on a thick iron plate which is positioned 


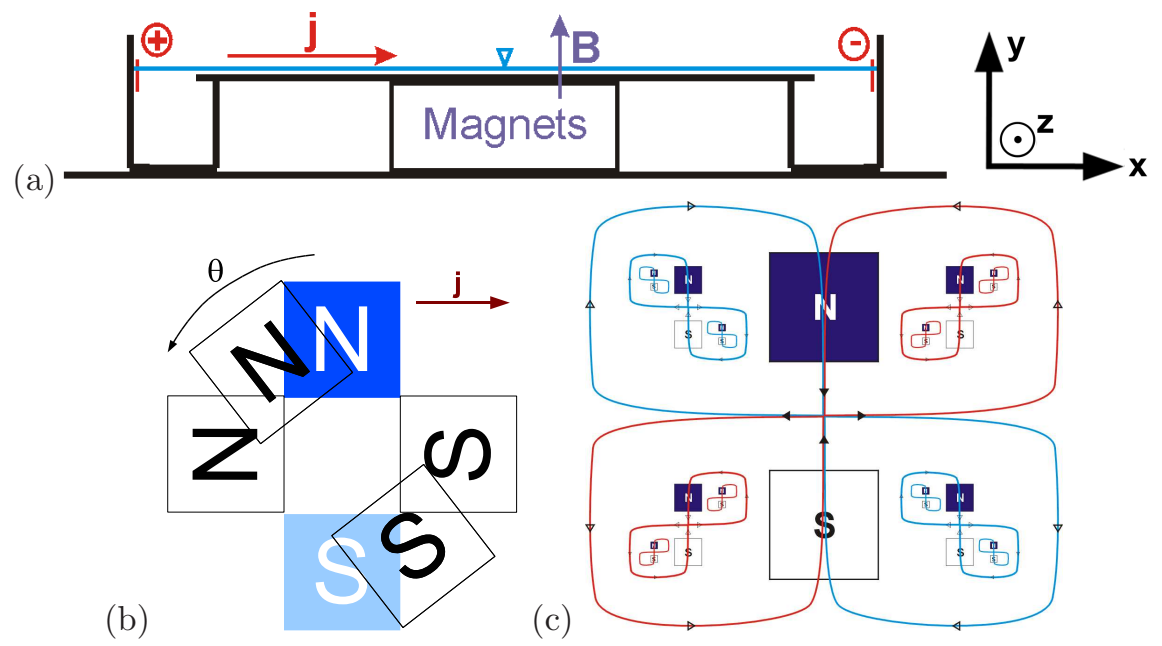

Figure 1: (a) Side view of the electromagnetic mixer rig, (b,c) Top-view (2D schematic) of the distribution of permanent magnets under the brine supporting wall: (b) single pair of magnets, for different angle $\theta$ and (c) multi-scale distribution. Current is from left to right.

at the centre of the tank, at about $3.7 \mathrm{~mm}$ from the brine. New real-time PIV measurements are performed using a $14 \mathrm{~Hz}$ camera $\left(2048 \times 2048\right.$ pixel $\left.^{2}, 14 \mathrm{bit}\right)$ and a picture frame of $441 \times 441 \mathrm{~mm}^{2}$. Pliolite DF01 $(100 \mu \mathrm{m}<$ diameter $<200 \mu \mathrm{m})$ is used to seed the flow and PIV post-processing is performed with an in-house code (iterative method with sub-pixel accuracy). The typical maximal displacements are about 16 pixels per frame and the smallest correlation window of the iterative process is of 16 pixels by 16 pixels. The characteristic values of the correlation coefficients of these measurements are about 0.9. We compute the PIV with a grid of $222 \times 222$ points. The corresponding distance between two velocity points is of 8 pixels. This gives more than $20 \times 20$ points above each magnets and a final correlation window size about 12 times smaller than the magnet dimensions. To extract the acceleration fields from this PIV data, which is well resolve in space and time, we use the PTVA algorithm [7] that we apply on a virtual Lagrangian tracking (the temporal integration is performed using 4th order Runge Kutta algorithm (RK4), with a time step adjusted to a quarter of the mesh size divided by the maximal velocity, and the spatial interpolation is performed using Lagrange polynomial of order 2). We should indicate here that we also did test a real tracking based on this PIV data, but no improvement was gained by using it as it mainly added a noise (when compared to virtual tracking) which could not be considered as small compared to the distance between two particles. A very large number of virtual particles are tracked so as to ensure that we get more than 18 positions in almost each window of $16 \times 16$ pixels $^{2}$ (which corresponds to the smallest scale of the PIV) before extracting the new grids, keeping a distance of 8 pixels between two grid points. The PTVA algorithm [7] is run with a $\Delta_{\text {target }}=32$ pixels which corre- 
sponds to two times the size of the smallest correlation windows. Consequently, the smallest eddies which can be resolved by our PTVA algorithm are about 5 pixels in diameter which is well smaller than the mesh size of the grids. This is a very extensive process requiring significant amount of CPU time, nevertheless we do prefer this approach (and filtering via Lagrangian integration and PTVA) rather than a direct derivation of the space and time resolved PIV data, as this improve the quality.

It should be noted that the description of the multi-scale experiments can be found in earlier papers $[23,7]$. Their quality is similar to the one just presented except that the acceleration data presented in the multi-scale section is extracted using real particle tracking and the PTVA algorithm.

\subsection{Simulations}

The electromagnetic forces model is based on the model proposed in [26, $21]$ using the analytical solution of [2]. The 3D distribution of the permanent magnets and an uniform distribution of the electrical current density along the $x$ axis are then used to compute the electromagnetic forces.

The equations of motion solved are the classical incompressible forced NavierStokes equations:

$$
\begin{gathered}
\frac{\partial \mathbf{u}}{\partial t}+(\mathbf{u} \nabla) \cdot \mathbf{u}=-\frac{1}{\rho} \nabla p+\nu \Delta \mathbf{u}+\frac{1}{\rho} \mathbf{f} \\
\nabla \cdot \mathbf{u}=0
\end{gathered}
$$

where $\mathbf{u}(\mathbf{x}, t)$ is the velocity vector, $\rho$ the constant density, $p(\mathbf{x}, t)$ the pressure field, $\nu$ the kinematic viscosity and $\mathbf{f}(\mathbf{x})$ is the electromagnetic forcing defined in section 2.1 .

Computations are carried out with a finite difference code, using 6th-order compact scheme for spatial discretisation and 3rd order Runge-Kutta scheme for time derivatives. Poisson equation is solved directly using Fourier decomposition. Extensive numerical tests have been conducted to minimise spatial and temporal discretisation errors. More details about the computation of the electromagnetic forces and the 3D numerical methods are given in [14]. For the single pair of magnets, the domain dimensions are $\left(L_{x}^{+}, L_{y}^{+}, L_{z}^{+}\right)=$ $(100,1,100)$ in the $x, y$ and $z$ directions respectively, and it is discretised on a mesh $\left(n_{x}, n_{y}, n_{z}\right)=(193,21,193)$. For the multi-scale forcing, the size of the computational domain is equal to $\left(L_{x}^{+}, L_{y}^{+}, L_{z}^{+}\right)=(240,1,240)$ in the $x$, $y$ and $z$ directions respectively, and it is discretised on a mesh $\left(n_{x}, n_{y}, n_{z}\right)=$ $(301,17,301)$.

\section{Flow topology using a single pair of magnets}

Experimental and numerical results show that the flow geometry and topology can be easily controlled by varying the position of a single pair of permanent magnets. The change of topology is performed by varying the geometry of the 


\begin{tabular}{c|ccccccc}
$\theta$ & $0^{\circ}$ & $15^{\circ}$ & $30^{\circ}$ & $45^{\circ}$ & $60^{\circ}$ & $90^{\circ}$ & $180^{\circ}$ \\
\hline$u_{r m s} \operatorname{Exp}$ & 1.74 & 1.89 & 1.93 & 2.1 & 2.09 & 1.98 & 2 \\
$u_{r m s} \operatorname{Sim}$ & 1.78 & 1.82 & 1.78 & 1.88 & 2 & 2.03 & 1.73 \\
$\sigma^{*} \operatorname{Exp}$ & $2.4 \%$ & $3 \%$ & $3.4 \%$ & $3.1 \%$ & $3.4 \%$ & $4.4 \%$ & $3.4 \%$ \\
$\sigma^{*} \operatorname{Sim}$ & $0.57 \%$ & $0.06 \%$ & $0.2 \%$ & $0.57 \%$ & $0.55 \%$ & $0.38 \%$ & $2.86 \%$ \\
$R e$ & 8 & 8 & 9 & 9 & 9 & 9 & 9 \\
$H a^{2}$ & 45 & 41 & 40 & 37 & 37 & 39 & 39 \\
geometry & $\mathcal{H}$ & $\mathcal{H}$ & $\mathcal{H}$ & $\mathcal{E}$ & $\mathcal{E}$ & $\mathcal{E}$ & $\mathcal{H}$
\end{tabular}

Table 1: Typical values and geometries obtained in the experiments and numerical simulations $(I=48.6 \mathrm{~mA})$. $u_{r m s}$ Sim are computed at $y^{+}=0.5$, values of $u_{r m s}$ are given in $\mathrm{mm} / \mathrm{s}$. The notations $\mathcal{H}$ and $\mathcal{E}$ corresponds to hyperbolic and elliptic stagnation point between the magnets, respectively.

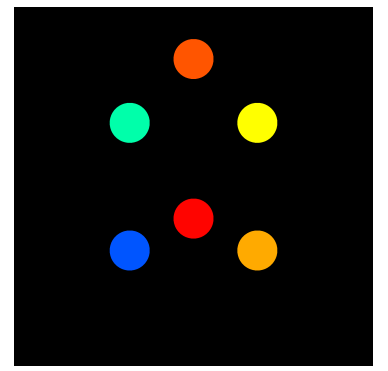

Figure 2: Initial distribution of the passive tracers used in our visualisations. Each color represent a different blob of tracers

forcing: the pair of square permanent magnets are rotated by an angle $\theta$, as illustrated in Fig. 1, where $\theta$ corresponds to the orientation of the magnets with respect to the direction normal to the electrical field. Different angles have been considered (Table 1 ), ranging from $0^{\circ}$ to $180^{\circ}$. For all cases, the Reynolds number, based on rms velocity and the thickness of the brine, $R e=\frac{u_{r m s} H}{\nu}$, is below 10, hence the flows are laminar. For all cases, the Hartmann number is larger than 10 and confirms that the forcing is strong enough to drive the flow.

In his seminal talk, Reynolds [19] did propose to use coloured bands to study fluid motion and introduced the importance of stretching and folding mechanisms in the process of mixing. Here, we use coloured blobs to first characterise the flows and discuss the associated stirring mechanism. Consequently, velocity fields and visualisations of passive scalar for three different angles are given in Fig. 3. The plots illustrate different flow topologies generated by single forcing. Visualisations of the dispersion of non-diffusive passive scalar, for which the initial distribution, similar for all cases, is given in Fig. 2 are used to describe qualitatively the different stirring properties of those flows.

For $\theta=0^{\circ}$, the brine is pumped above the magnets in the $z$-direction (flow directions above the magnets are indicated by red arrows for all cases, Fig. 


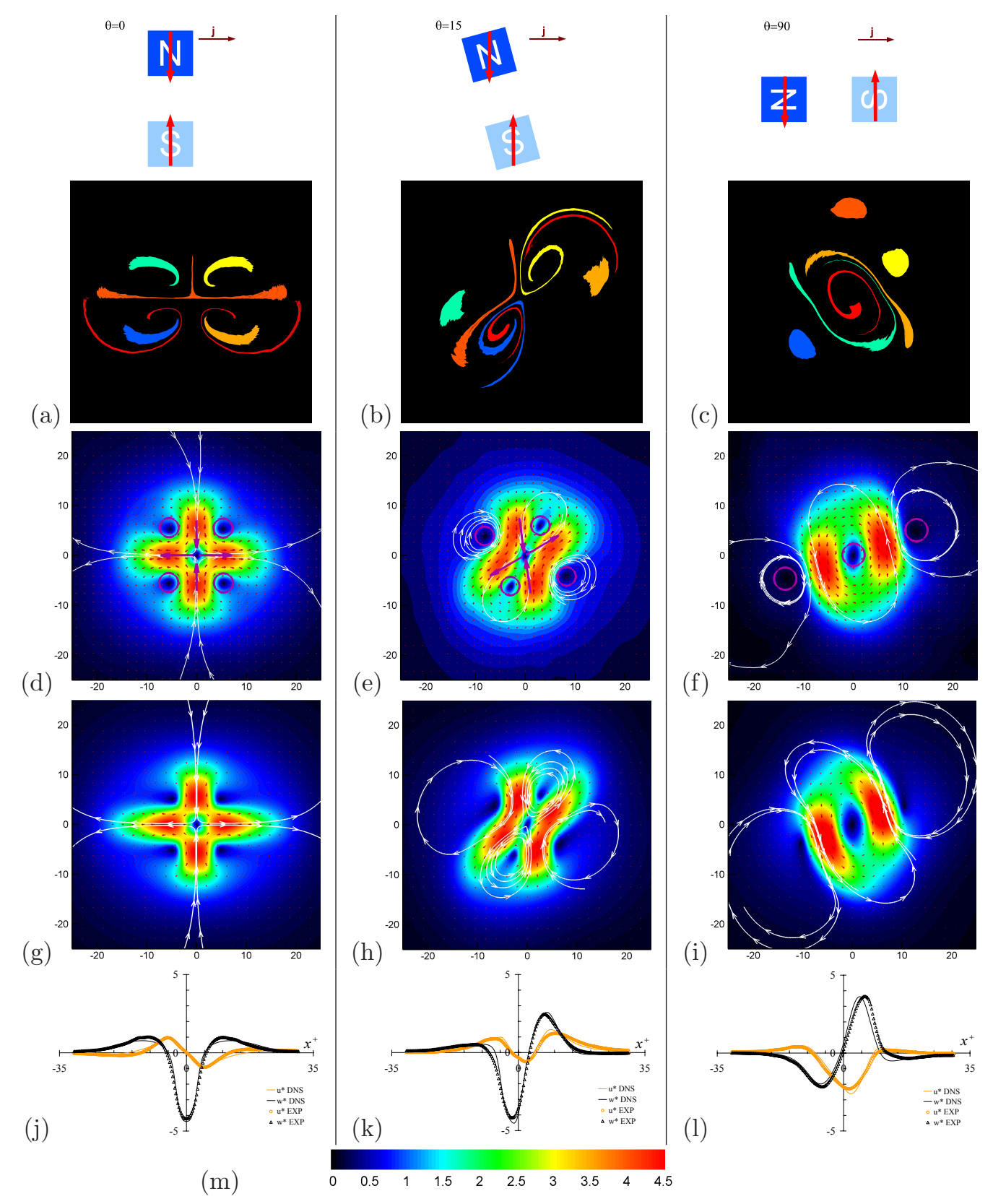

Figure 3: (a,b,c) Visualisations of scalar dispersion (initial distribution given in Fig. 2), (d,e,f) Isocontours of velocity intensity $\left\|u^{*}\right\|$, from PIV/PTVA measurements, $(\mathrm{g}, \mathrm{h}, \mathrm{i})$ same as (d,e,f) using 3D DNS data, at $y^{+}=0.5$. ( $\left.\mathrm{j}, \mathrm{k}, \mathrm{l}\right)$ Comparison of experimental and numerical velocity profiles at $z^{+}=6.66$. $(\mathrm{m})$ scale of $\left\|u^{*}\right\|$ maps. Streamlines are plotted with white lines, and small arrows show the flow direction. The large arrows and circles indicate particular flow features, i.e. the flow around hyperbolic and elliptical stagnation points. 
3, top row), generating two opposite colliding jets. These two jets generate, in between the magnets, an hyperbolic stagnation point (noted $\mathcal{H}$ in Table 1) with orthogonal eigen-directions, as indicated by purple arrows in opposite directions on Fig. 3d). The domain close to the stagnation point is characterised by high strain and low vorticity, with $\mathbf{u} \simeq \lambda \mathbf{x}$ and $\mathbf{w} \simeq-\lambda \mathbf{z}$ in the eigenframe where $\pm \lambda$ are the eigen values of the Jacobian matrix $J_{[i, j]}=\left[\frac{\partial u_{i}}{\partial x_{j}}\right]$, e.g. [16]. The corresponding stirring mechanism are illustrated by the tracer visualisations (Fig. 3a). The time history of the dispersion of the top blob of dye (orange color online) is the following: the blob is pumped above the top magnet, then stretched around the hyperbolic stagnation point, and then pushed in two opposite directions in the horizontal axis of symmetry of the flow ( $c f$. movie mov_0.avi).

For $\theta=15^{\circ}$, the two colliding jets are not strictly opposite, they thus generate an eight shape (inclined with respect to the $z$-direction). This is illustrated by the velocity fields and the streamlines (Fig. 3e). The stagnation point remains hyperbolic, but the eigen-directions are no longer orthogonal (arrows on Fig.3e). In this case, the domain close to the stagnation point is an area with high strain and the two parts between the eigen-directions (within the eight) are also with a high vorticity. Tracer visualisations shown on Fig.3(b) illustrates the stirring mechanism associated with this flow geometry. The scalar dispersion of the blob of dye initially close to the centre (red color online) is taken as example. The tracer is initially pumped above the bottom magnet towards the hyperbolic stagnation point, then it splits into two streams and each part is trapped inside the eight pattern ( $c f$. movie mov_15.avi).

For $\theta=90^{\circ}$, the two jets are parallel: they trapped each other to form a recirculation. The velocity fields given in Fig. 3(f) show how the two opposite and distant pumping generate and sustain this main recirculation. In this case, the forcing generates an elliptical stagnation point $(\mathcal{E})$ in between the two magnets. The tracers (Fig. 3c) are put in motion forming an oval recirculation ( $c f$. movie mov_90.avi). Nevertheless, we can notice that the lamination and folding of the tracers in this configuration is weaker than for the two previous cases.

As the initial distribution of scalar is the same in all cases (Fig. 2), Fig. 3a, $\mathrm{b}$ and $\mathrm{c}$ can be directly compared: they illustrate different stirring mechanisms. Each of the configurations possesses specific stretching and folding properties, as well as different symmetries. Geometries similar to case $\theta=0^{\circ}$ is of interest for stretching, the geometry corresponding to $\theta=15^{\circ}$ is of interest for stretching, lamination and folding, and that geometry of the type observed for $\theta=90^{\circ}$ is more interesting to break the symmetries and rotate/swap the flow.

The type $(\mathcal{H}$ or $\mathcal{E})$ of stagnation point is reported for all cases in Table 1. The flow topology changes between $30^{\circ}$ and $45^{\circ}$. More refined experiments (not reported here) localize the change in geometry from hyperbolic to elliptic configuration between $30^{\circ}$ and $32.5^{\circ}$ and from elliptic to hyperbolic configuration between $90^{\circ}$ and $120^{\circ}$. It is also worth noticing that the transition from $\mathcal{H}$-type $\left(\theta=0^{\circ}\right)$ to $\mathcal{E}$-type $\left(\theta=90^{\circ}\right)$ stagnation point produces unsteady flow, the strongest unsteadiness being observed for larger intensity of the forcing than 
that presented here. This unsteady regime depends also on the thickness of the brine.

Velocity fields are presented for both numerical simulations and experiments (Fig. 3d,e,f against Fig. 3g,h,i). Results for experiments and simulations are similar, both for flow geometry and intensity. Even if the results are close, some differences can still be noted. A comparison of velocity profiles is also given for $z^{+}=6.66$, to assess the accuracy of the electromagnetic forcing model. A global quantification of the differences is obtained by computing the difference between the numerical and the experimental flow field (not shown here), and then averaging over the entire flow field. The global error varies between $8 \%$ and $14 \%$ for the considered angles. It is also important to point out that even if the electromagnetic forces in the case $\theta=180^{\circ}$ are opposite to that of $\theta=0^{\circ}$, the resulting flows are not symmetric and present significant differences, both in experiments and in simulations. These differences indicate that even if the flow is at low Reynolds number, the non-linear terms (inertial terms) should be taken into account to capture correctly the flow physics.

In addition, the values of the temporal standard deviation are given in table $1:$

$$
\sigma^{*}=\frac{\left\langle\sqrt{\overline{(u(x, y)-\overline{u(x, z)})^{2}}}\right\rangle_{x, z}}{u_{r m s}}
$$

where - indicates a time averaged value, and $\langle\cdot\rangle_{x, z}$ indicates a value averaged in $x$ and $z$-directions. Values of $\sigma^{*}$ obtained for the experiments (PIV/PTVA processing) are low and well under the typical PIV measurement noise of 0.2 pixel per frame which would lead to $\sigma_{\text {noise }}^{*} \simeq 5.6 \%$. Also, the values obtained for the numerical simulations are generally one order of magnitude lower, showing that the present flows are weakly time dependent and could be considered as quasi-steady.

Finally, it should be indicated that the time for the flow to converge to a quasi-steady solution depends on the considered angle. It is fast (30s, real time) for $0^{\circ}$ and $180^{\circ}$, whilst for other angles a quasi-steady state is reached after a much longer time, as high as $120 \mathrm{~s}$ for $\theta=90^{\circ}$.

Fig. 4 shows the comparison of acceleration field between simulation and experiments. Such results are more sensitive to errors than velocity, hence it is a good way to compare the quality of our experiments and simulations. Fig. 4 shows that the main patterns of the acceleration are similar between experiments and simulations whilst the small differences observed in the velocity profiles are amplified for the acceleration profiles. Nevertheless, the global quantification of errors on the acceleration field gives an average error under $10 \%$ (for all the angle considered) which is still a good general agreement between simulations and experiments.

The geometry of the acceleration fields also varies with the angle $\theta$. It is clear that the geometry and topology of the acceleration fields given in Fig. 4 are strongly different according to the nature of the main designed stagnation point in between the magnets, i.e. hyperbolic or elliptic. It should also be noticed that the distribution of sinks and sources of acceleration follows the structure of the 
$\theta=0$

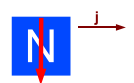

(a)

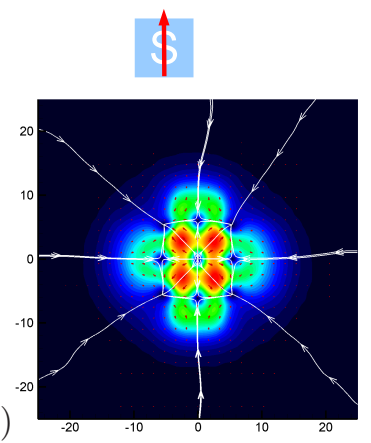

(d)

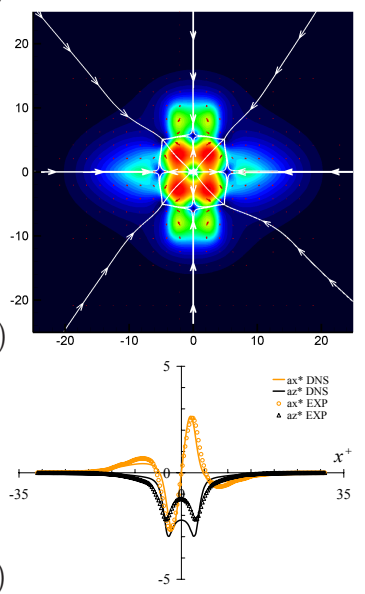

(j)

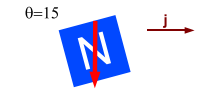

(b)

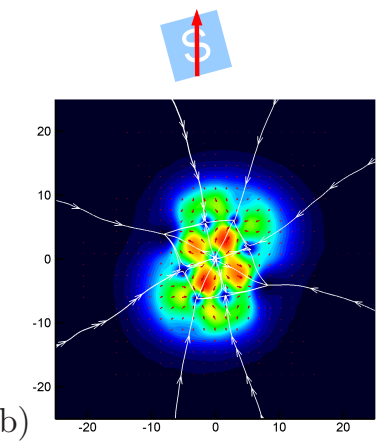

(e)

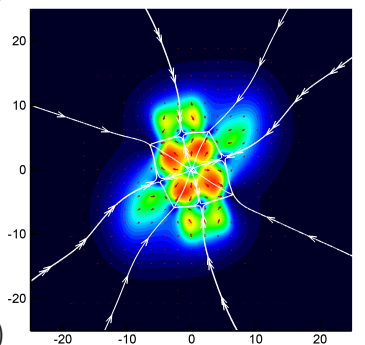

(h)

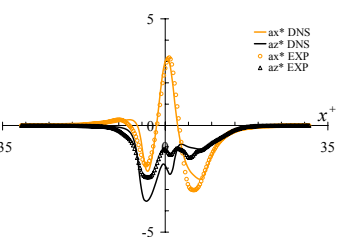

$\theta=90 \stackrel{\mathrm{j}}{\longrightarrow}$

$2 z$

(c)

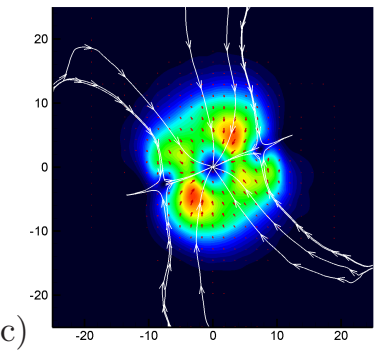

(f)

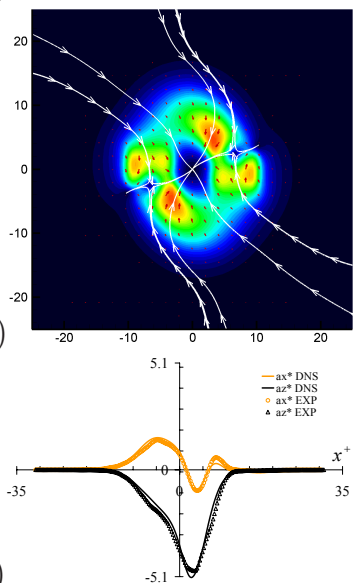

Figure 4: (a,b,c) and acceleration intensity $\left\|a^{*}\right\|$ respectively from PIV/PTVA measurements and $3 \mathrm{D}$ DNS at $y^{+}=0.5$. $(\mathrm{g}, \mathrm{h}, \mathrm{i})$ Acceleration profiles at $z^{+} \simeq 6.66$ for experiments and simulations. (j) colour-scale for $\left\|a^{*}\right\|$ maps. Acceleration-lines are plotted with white lines, arrows indicate the direction of the Lagrangian acceleration. 


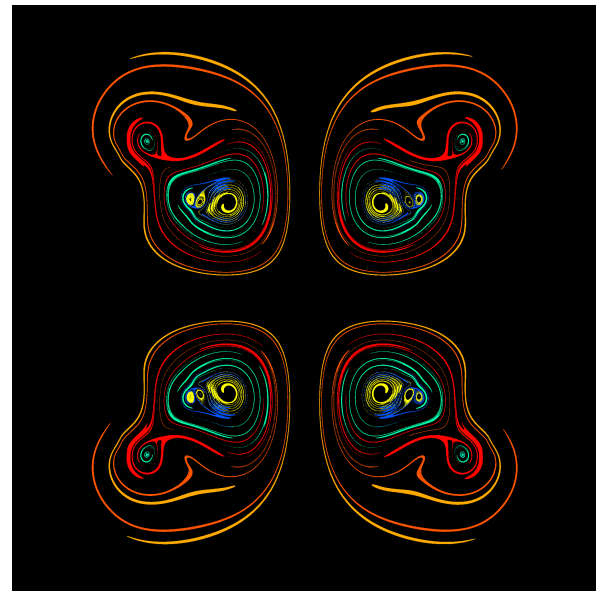

(a)

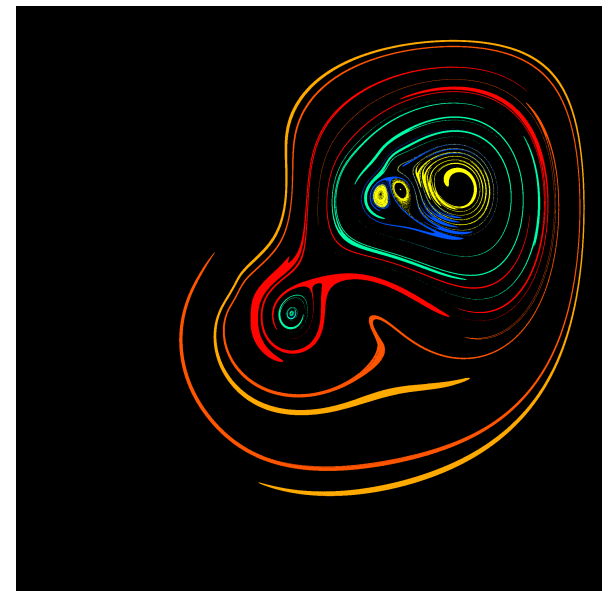

(b)

Figure 5: visualisations (from DNS) in large (a) and small (b) frames of a flow forced by a multi-scale distribution of pair of magnets. Illustration after 9 large-scale turnover times.

flow. Hyperbolic stagnation points correspond to sources of acceleration with $\nabla \cdot \mathbf{a}>0$ and $\nabla \cdot \mathbf{a}$ is locally maximum at these points. Acceleration streamlines are emanating from these points and are connected to sinks of acceleration corresponding to elliptical stagnation points, where $\nabla \cdot \mathbf{a}<0$ and $\nabla \cdot \mathbf{a}$ is locally minimum. These connections between sources and sinks are observed and design an acceleration web of the flows which works differently than streamlines, where elliptical stagnation points are not directly connected to hyperbolic stagnation points via velocity streamlines.

\section{Flow topology using multi-scale forcing}

A complementary extension is to apply the control of the flow geometry in a multi-scale pattern. The objective of this extension is to target and control specific and complementary length- and time-scales to stir the flow. This multi-scale design possess turbulent-like features and statistics [23, 24] whilst it is imposed by an ordered principle in laminar condition. This multi-scale approach is briefly illustrated here so as to support the concept of flow design with more than one scale. It also highlight the good complementarity and agreement between the present experimental and numerical results; for more details about this specific configuration, see [24, 14].

Fig. 5 illustrates the control of the flow geometry and topology using multiscale forcing. This forcing is designed to insert the structures previously observed within each other with decreasing scales. The multi-scale structure is generated by a combination of pair of magnets with an orientation of $\theta=0^{\circ}$ (Fig. 1 and [23] for more details). Similar to the case with one pair of magnets, 
the brine is pumped over each pair of magnets in opposite directions. This leads to a distribution of colliding jets and induced $\mathcal{H}$ and $\mathcal{E}$ points along a multi-scale pattern. Each colliding jets also produce a multi-scale distribution of strain rates according to the size of the pair of magnets and the corresponding intensity of the strain rate. Importantly, this combination of different stirring and stretching within each flow structures (or eight) is amenable to statically produce a Richardson-like dispersion which is characteristic of turbulent flows whilst these flows are steady and laminar (for more details see [24]). See also the movie multi-scale.avi, for a temporal illustration of this multi-scale stirring.

Fig. 6 shows a global, qualitative, comparison of both experimental and numerical velocity fields. The same general flow topology is observed in both cases, at almost every forcing scales, and this comparison is completed by comparing experimental and numerical velocity profiles extracted along an axis crossing all forcing scales (shown by dashed line on the velocity fields). Simulations and experiments are highly correlated with errors of about $13 \%$, which clearly confirms the validity of the $3 \mathrm{D}$ DNS to reproduce faithfully the experiments. In addition, it shows the significant improvement provided by those $3 \mathrm{D}$ simulations, compared to previous 2D simulations, [8]. As shown by [14], this significant improvement is due to the use of a more detailed 3D model for the description of the electromagnetic forces and to a better modeling of the wall-normal shear which is explicitly resolved in $3 \mathrm{D}$ simulations, whilst the flow can still be considered as quasi-two-dimensional in terms of energy.

3D DNS data are compared with the acceleration field measured experimentally [7] using Particle Tracking Velocimetry and Accelerometry (PTVA), on Fig. 6. The same multi-scale structure of the acceleration field is observed in both experiments and numerical simulations. Profile of the two acceleration components, extracted along the same axis as those used for velocity comparisons (illustrated by dashed lines on the accelerations fields), are shown on Fig. 6. These acceleration profiles confirm even further the good agreement between 3D simulation and experiment. Some differences, as for example the two black peaks, are coming from a slight displacement of magnets positions in experiments from the initial design (about $1 \mathrm{~mm}$ for a tank of $1700 \mathrm{~mm}$ ). A more complete validation of acceleration components of these multi-scale flows can be found in [14].

\section{Conclusion}

Experimental and numerical results presented in this paper show that we can design and control the flow geometry/topology using body forces. This can be performed by varying the orientation of a single pair of permanent magnets with respect to the electrical current. By tracking passive scalars, it is shown that these flows possess different stirring properties according to the selected geometries. These first results are encouraging as they show that complementary basic flows (building blocks) with various stretching, folding and swapping properties can be designed at will. In the context of mixing, temporal and 


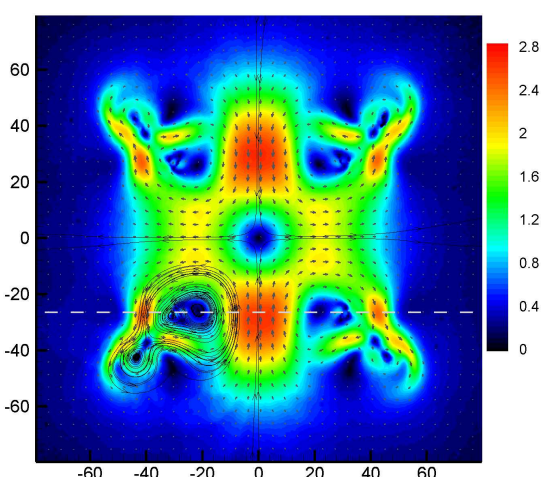

(a)

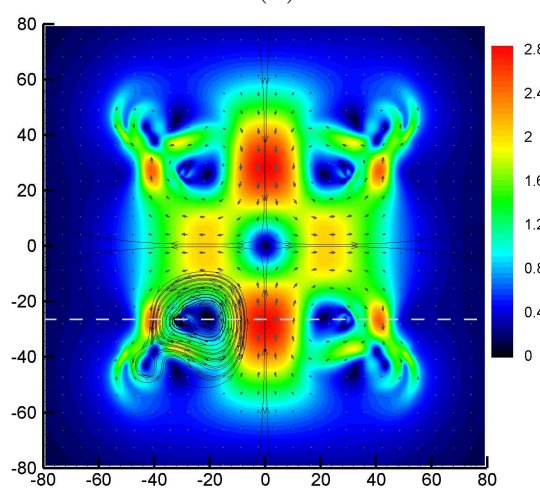

(c)

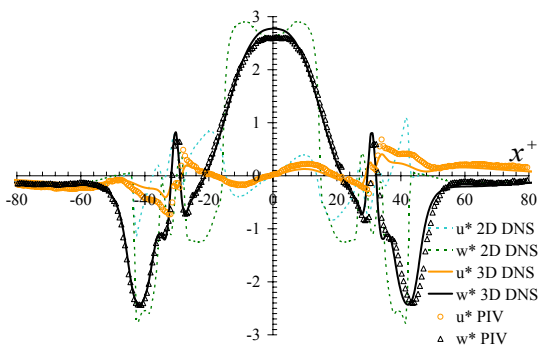

(e)

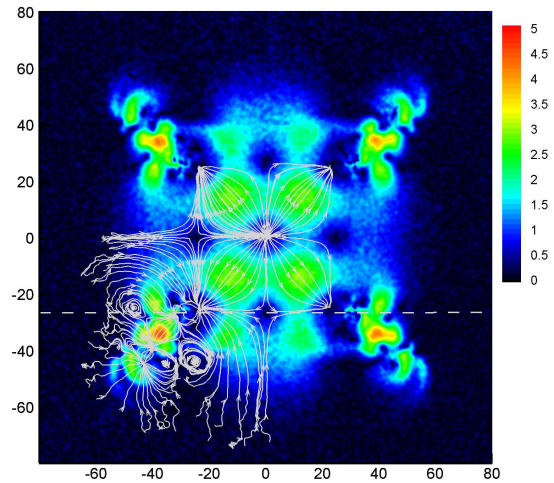

(b)

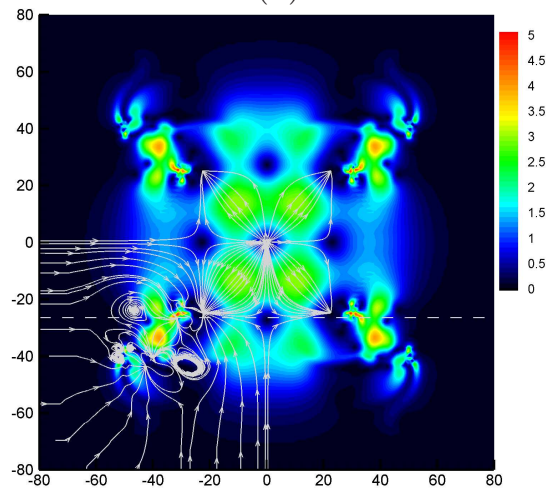

(d)

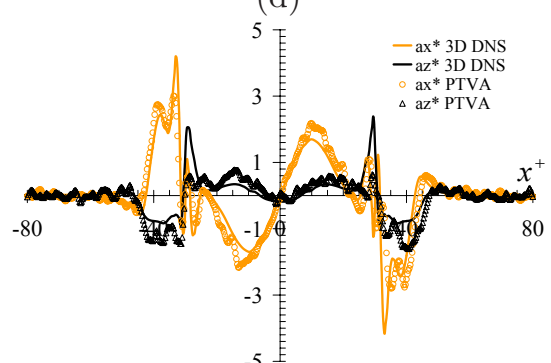

(f)

Figure 6: (a,c) Velocity fields, from PIV measurements (a) [23] and 3D DNS at $y^{+}=0.5$ for $I=0.3 \mathrm{~A}(\mathrm{c})$. Streamlines are plotted with black lines, arrows indicate the flow direction. (b,d) Acceleration field from (top) PTVA measurement [7] and 3D DNS acceleration field at $y^{+}=0.5, \mathrm{I}=0.53 \mathrm{~A}$. The scales (color online) present the velocity, $\left\|\mathbf{u}^{*}\right\|$, and Lagrangian acceleration, $\left\|\mathbf{a}^{*}\right\|$, intensities. (e) $u^{*}$ and $w^{*}$ velocity profiles for $y^{+}=0.5$ and $I=0.3 \mathrm{~A}$, along the axis $z^{+}=-26.4$. 3D DNS results are represented with solid lines and PIV results with symbols. 2D DNS [8] results are in dashed lines. (f) $a_{x}^{*}$ and $a_{z}^{*}$ acceleration profiles for $z^{+}=-26.4$. DNS results are plotted with solid lines and PIV results with symbols, $\mathrm{I}=0.53 \mathrm{~A}$. 
spatial combination of these basics structures should be key. For example, combination of hyperbolic and elliptic configurations should allow to perform in the laboratory controlled tendril-whorl flows [13]. The design and control of flow geometries (and topologies) is also extended to multi-scale flows by using multi-scale distribution of the body forces. This level of control obtained by using electromagnetic forcing allows to design very conceptual and fundamental experiments as those presented in this paper.

A good agreement between the 3D simulations and experiments is found for both single and multi-scale forcing. This good agreement is also found on both velocity and Lagrangian acceleration. This reinforces the confidence in both simulations and experiments as no parameters have been tuned in numerical simulations to make them match the experiments, which have been obtained using different techniques. Also, the complementarity of the information which is gain from the access to velocity and accelerations fields, such as connections of topological features, is expected to be important for flow design, control and modeling. Its characterization is part of the authors current and future research.

In addition to velocity and acceleration which are obtained via PIV, PTVA and 3D DNS, the authors are currently investigating the mixing properties of these flows using Light Induced Fluorescence (LIF) measurements, in order to extract the time evolution of scalar variance and probability distribution functions. The authors are then able to perform and analyse complementary experiments (e.g. PIV, PTVA, LIF) and numerical simulations. Those tools can thus be used to analyse the mixing properties of the designed flows, along with the distribution of the acceleration fields and working forces, in order to design, control and optimize these electromagnetic mixers.

\section{Acknowledgments}

We acknowledge the Marie Curie Multi-Partner European Training Site on Environmental Turbulence, the EPSRC, The Royal Society, the Turbulence Mixing and Flow Control group and Dr. Hascoët.

\section{References}

[1] Akkermans, R. A. D., Cieslik, A. R., Kamp, L. P. J., Trieling, R. R., Clercx, H. J. H., Heijst, G. J. F. v., 2008. The three-dimensional structure of an electromagnetically generated dipolar vortex in a shallow fluid layer. Phys. Fluids 20 (11), 116601.

[2] Akoun, G., Yonnet, J., 1984. 3d analytical calculation of the forces exerted between two cuboidal magnets. IEEE Transaction on magnetics 20 (5), 1962-1964.

[3] Aref, H., 1984. Stirring by chaotic advection. J. Fluid. Mech. 143, 1-21. 
[4] Arratia, P. E., Gollub, J. P., 2006. Predicting the progress of diffusively limited chemical reactions in the presence of chaotic advection. Phys. Rev. Lett. 96 (2), 024501.

[5] Breuer, K. S., Park, J., Henoch, C., 2004. Actuation and control of a turbulent channel flow using lorentz forces. Phys. Fluids 16 (4), 897-907.

[6] Dukure, R., Lielausis, O., 1962. Experimental investigation of the effect of electromagnetical forces upon flow around bodies in electrolyte. Problems of Magnetohydrodynamics and Plasmadynamics (Voprosi Magnitnoi Gidrodinamiki i Dinamiki Plazmi) Riga, 647-650.

[7] Ferrari, S., Rossi, L., 2008. Particle tracking velocimetry and accelerometry (ptva) measurements applied to quasi-two-dimensional multi-scale flows. Exp. Fluids 44 (6), 873-886, 10.1007/s00348-007-0443-7.

[8] Hascoet, E., Rossi, L., J.C., V., 2008. Multi-scale flow control for efficient mixing: simulation of electromagnetically forced turbulent-like laminar flows. IUTAM Symposium on Flow Control and MEMS, 273-277.

[9] Henoch, C., Stace, J., 1995. Experimental investigation of a salt water turbulent boundary layer modified by an applied streamwise magnetohydrodynamic body force. Phys. Fluids 7 (6), 1371-1383.

[10] Jullien, M.-C., Castiglione, P., Tabeling, P., 2000. Experimental observation of batchelor dispersion of passive tracers. Phys. Rev. Lett. 85 (17), 3636 .

[11] Kenjeres, S., 2008. Electromagnetic enhancement of turbulent heat transfer. Phys. Rev. E 78 (6), 066309.

[12] Kenjeres, S., Verdoold, J., Tummers, M., Hanjalic, K., Kleijn, C., 2008. Numerical and experimental study of electromagnetic driven vortical flows. ETMM7, 6.

[13] Khakhar, D. V., Rising, H., Ottino, J. M., 1986. Analysis of chaotic mixing in two model systems. J. Fluid. Mech. 172, 419-451.

[14] Lardeau, S., Ferrari, S., Rossi, L., 2008. Three-dimensional direct numerical simulation of electromagnetically driven multiscale shallow layer flows: Numerical modeling and physical properties. Phys. Fluids 20 (12), 127101.

[15] Nosenchuck, D., Brown, G., 1993. Discrete spatial control of wall shear stress in a turbulent boundary layer. in Near-Wall Turbulent Flows, edited by R. So C. Speziale, and B. Launder (Elsevier Science, New York), 689 698.

[16] Ottino, J. M., 1989. The Kinematics of mixing: stretching, chaos and transport. Cambridge University Press. 
[17] Ouellette, N. T., Gollub, J. P., 2008. Dynamic topology in spatiotemporal chaos. Phys. Fluids 20 (6), 064104.

[18] Perry, A. E., Chong, M. S., 1987. A description of eddying motions and flow patterns using critical-point concepts. Ann. Rev. Fluid. Mech. 19, 125-155.

[19] Reynolds, O., 1893. Study of fluid motion by means of coloured bands. Proceedings of the Royal Institution of Great Britain XIV, 129-138.

[20] Rivera, M. K., Ecke, R. E., 2005. Pair dispersion and doubling time statistics in two-dimensional turbulence. Phys. Rev. Lett. 95 (19), 194503.

[21] Rossi, L., 2001. Control electromagnetique d'ecoulement en eau de mer. $\mathrm{PhD}$ theses Universite Joseph Fourier Grenoble.

[22] Rossi, L., Thibault, J.-P., 2002. Investigation of wall normal electromagnetic actuator for seawater flow control. Journal of Turbulence 3, N5.

[23] Rossi, L., Vassilicos, J. C., Hardalupas, Y., 2006. Electromagnetically controlled multi-scale flows. J. Fluid. Mech. 558, 207-242.

[24] Rossi, L., Vassilicos, J. C., Hardalupas, Y., 2006. Multiscale laminar flows with turbulentlike properties. Phys. Rev. Lett. 97 (14), 144501.

[25] Tabeling, P., 2002. Two-dimensional turbulence: a physicist approach. Phys. Rep. 362 (1), 1-62, 10.1016/S0370-1573(01)00064-3.

[26] Thibault, J.-P., Rossi, L., 2003. Electromagnetic flow control: characteristic numbers and flow regimes of a wall-normal actuator. J. Phys. D: Appl. Phys. 36 (20), 2559.

[27] Voth, G. A., Saint, T. C., Dobler, G., Gollub, J. P., 2003. Mixing rates and symmetry breaking in two-dimensional chaotic flow. Phys. Fluids 15 (9), 2560-2566.

[28] Weier, T., Gerbeth, G., Mutschke, G., Lielausis, O., Lammers, G., 2003. Control of flow separation using electromagnetic forces. Flow, Turbulence and Combustion 71 (1), 5-17, 10.1023/B:APPL.0000014922.98309.21.

[29] Wiggins, S., Ottino, J. M., 2004. Foundations of chaotic mixing. Philosophical Transactions of the Royal Society of London. Series A: Mathematical, Physical and Engineering Sciences 362 (1818), 937-970.

[30] Wolf, A., Brown, M., Prentiss, P., 1982. Concentrative properties of aqueous solutions: conversion tables. CRC Handbook of Chemestry and Physics, CRC Press. 